\title{
Are there biological differences between screen-detected and interval colorectal cancers in the English Bowel Cancer Screening Programme?
}

\begin{abstract}
Elizabeth Walsh ${ }^{1}$, Colin J Rees ${ }^{2,3}$, Michael Gill ${ }^{2}$, Clare E Parker ${ }^{2}$, Roisin Bevan ${ }^{2}$, Sarah L Perry ${ }^{1}$, Yvonne Bury $^{4}$, Sarah Mills ${ }^{5}$, D Michael Bradburn ${ }^{5}$, Michael Bramble ${ }^{3}$ and Mark A Hull ${ }^{\star, 1,6}$

${ }^{1}$ Leeds Institute of Biomedical and Clinical Sciences, University of Leeds, St James's University Hospital, Leeds LS9 7TF, UK; ${ }^{2}$ South Tyneside NHS Foundation Trust, South Tyneside District Hospital, South Shields NE34 OPL, UK; ${ }^{3}$ School of Medicine, Pharmacy and Health, University of Durham, Durham, UK; ${ }^{4}$ Newcastle upon Tyne Teaching Hospitals NHS Foundation Trust, Royal Victoria Infirmary, Newcastle upon Tyne NE1 4LP, UK; ${ }^{5}$ Northumbria Healthcare NHS Foundation Trust, North Tyneside General Hospital, North Shields NE29 8NH, UK and ${ }^{6}$ Centre for Digestive Diseases, St James's University Hospital, Leeds Teaching Hospitals NHS Trust, Leeds LS9 7TF, UK
\end{abstract}

Background: We measured biomarkers of tumour growth and vascularity in interval and screen-detected colorectal cancers (CRCs) in the English Bowel Cancer Screening Programme in order to determine whether rapid tumour growth might contribute to interval CRC (a CRC diagnosed between a negative guaiac stool test and the next scheduled screening episode).

Methods: Formalin-fixed, paraffin-embedded sections from 71 CRCs (screen-detected 43, interval 28) underwent immunohistochemistry for CD31 and Ki-67, in order to measure the microvessel density (MVD) and proliferation index (PI), respectively, as well as microsatellite instability (MSI) testing.

Results: Interval CRCs were larger $(P=0.02)$ and were more likely to exhibit venous invasion $(P=0.005)$ than screen-detected tumours. There was no significant difference in MVD or PI between interval and screen-detected CRCs. More interval CRCs displayed MSI-high (14\%) compared with screen-detected tumours (5\%). A significantly $(P=0.005)$ higher proportion $(51 \%)$ of screen-detected CRC resection specimens contained at least one polyp compared with interval CRC (18\%) resections.

Conclusions: We found no evidence of biological differences between interval and screen-detected CRCs, consistent with the low sensitivity of guaiac stool testing as the main driver of interval CRC. The contribution of synchronous adenomas to occult blood loss for screening requires further investigation.

The NHS Bowel Cancer Screening Programme (BCSP), based on biennial guaiac-based faecal occult blood testing (gFOBt), was introduced in England in 2006 with the aim of reducing mortality from colorectal cancer (CRC) by 16\% (West et al, 2008). Interval CRC (defined as a CRC diagnosed within the 2-year window between a negative gFOBt episode and the next scheduled screening episode) is a well-recognised outcome in all studies of population-based gFOBt programmes and accounts for $30-60 \%$ of all CRC diagnoses in a screened population depending on the screening round (Morris et al, 2012). Interval CRCs are more

*Correspondence: Professor MA Hull; E-mail: M.A.Hull@leeds.ac.uk

Received 27 January 2016; revised 27 April 2016; accepted 29 April 2016; published online 24 May 2016

(c) 2016 Cancer Research UK. All rights reserved 0007 - 0920/16 
common in women and occur more frequently in the proximal colon (proximal to the splenic flexure) compared with screendetected CRCs (Gill et al, 2012; Steele et al, 2012).

It has been assumed that the majority of interval CRCs represent missed lesions related to false-negative gFOBt or missed colorectal neoplasia at colonoscopy. However, an alternative explanation is that differences in the biology of individual tumours allow rapid interval CRC growth between biennial screening episodes. We recently reported that patients with a screen-detected CRC have improved survival compared with individuals with a stage-matched interval CRC (Gill et al, 2014). Although lead-time bias cannot be excluded, these data further support the hypothesis that interval CRCs are biologically more aggressive and/or have less propensity to bleed (explaining the lack of gFOBt positivity). Moreover, interval CRCs in colonoscopic screening programmes are more likely to demonstrate microsatellite instability (MSI) (Sawhney et al, 2006), a feature associated with accelerated colorectal carcinogenesis in the setting of Lynch syndrome (Rubenstein et al, 2015).

Therefore, we compared biomarkers of tumour growth and vascularity in interval and screen-detected CRCs in order to determine whether tumour factors explain whether a given CRC is detected by a gFOBt screening episode or is diagnosed between screening episodes.

\section{MATERIALS AND METHODS}

Patient and CRC cohort. Approval was obtained from the London-Central Research Ethics Committee. A random sample of CRC cases were taken from The Northern Region Colorectal Cancer Audit Group (NORCCAG) cohort which had been previously used to determine differences between screen-detected (from the first, prevalent screening round) and interval CRCs (Gill et al, 2012). The CRCs were all diagnosed from 1 April 2007 to 31 March 2010 in the patients aged 60-69 years. To determine screening status, the cohort was cross-referenced with the regional BCSP database to gather information on gFOBt invitations and results, screening appointments and colonoscopies. An interval cancer was defined as a CRC, which was diagnosed between a negative gFOBt screening episode and before the next scheduled screening episode. A screen-detected CRC was defined as a case diagnosed through the English BCSP. This study did not include post-colonoscopy CRCs.

Archival formalin-fixed, paraffin-embedded CRC specimens and the corresponding anonymised histopathology report were obtained from three hospitals (North Tyneside General Hospital, South Tyneside General Hospital and Royal Victoria Infirmary, Newcastle upon Tyne, UK). A ratio of 2-3 screen-detected CRCs to one interval CRC was maintained across the study cohort in order to mirror the ratio of screen-detected to interval CRC cases observed in the full NORCCAG cohort. The following data were collected from the histopathology report, with all investigators blinded as to whether each CRC was screen-detected or an interval tumour; site of the CRC in the colorectum (proximal or distal to the splenic flexure), size, ulcerative morphology, Dukes' Stage, histological differentiation grade, draining lymph node involvement, presence of additional polyps, extramural venous invasion, lymphatic invasion. All analyses were performed blinded to the categorisation (screen-detected $v s$ interval) of the CRC specimen.

A sample size of 70 CRCs was chosen on the basis of a previous CD31 microvessel density (MVD) angiogenesis study, which reported a relationship between tumour MVD and clinical outcomes in primary CRC patients (Rajaganeshan et al, 2007).

Tumour histological analysis. CRC blocks were sectioned ( $5 \mu \mathrm{m}$ thickness) using a Leica RM2255 microtome (Milton Keynes, UK).
Sections were mounted on Thermo Scientific Superfrost Plus slides (Waltham, MA, USA) and left to dry for $24 \mathrm{~h}$ at $37^{\circ} \mathrm{C}$. A haematoxylin and eosin-stained section from each block was prepared and used to identify the orientation of the tissue including the luminal border of the tumour. For cases with more than one CRC block, a single block was chosen for further analysis based on subjective analysis of each haematoxylin and eosin section (at $\times 100$ magnification) for the presence of the highest-quality malignant tissue with orientation perpendicular to the luminal surface such that the maximum amount of luminal (often eroded) surface was available for vascular 'hotspot' counting (see below).

Immunohistochemistry for CD31. After dewaxing in xylene and rehydration in an ethanol series, slides were washed in running tap water. The slides were heated for $10 \mathrm{~min}$ in $10 \mathrm{~mm}$ citric acid buffer ( $\mathrm{pH}$ 6.0) using a $900 \mathrm{~W}$ microwave, left to cool for $20 \mathrm{~min}$, then placed under running tap water. Slides were added to $0.3 \%(\mathrm{v} / \mathrm{v})$ hydrogen peroxide solution in $100 \%$ methanol for $10 \mathrm{~min}$. They were then rinsed in tap water for $5 \mathrm{~min}$. Non-specific binding was blocked with Invitrogen (Thermo Fisher Scientific, Waltham, MA, USA) antibody diluent and then a 1:20 dilution of mouse monoclonal anti-CD31 (clone JC70A (Dako UK Ltd, Ely, UK) in antibody diluent was added for $1 \mathrm{~h}$ at room temperature. Following washes with tris-buffered saline plus $0.025 \%(\mathrm{v} / \mathrm{v})$ Tween 20 and tris-buffered saline alone, immunoreactivity was visualised using a mouse EnVision detection kit and counterstained with haematoxylin before mounting with DPX (Sigma-Aldrich, Dorset, UK).

Microvessel density analysis. Image analysis was performed by one individual (EW) using the Nikon Eclipse (Nikon UK Ltd., Kingston upon Thames, UK) E1000 microscope and NIS-Elements software (Nikon UK Ltd.). Three vascular 'hotspots' were identified on each slide. A 'hotspot' was defined as a high-power field $(\times 200$ magnification; $0.28 \mathrm{~mm}^{2}$ ) contained within a $\times 100$ magnification field that included luminal tumour surface (to ensure that the surface vasculature that was analysed is the most relevant to luminal blood loss), which contained the maximum number of CD31-positive vascular structures. The MVD in vessels per $\mathrm{mm}^{2}$ was determined. If a section was deemed to have less than three vascular 'hotspots', the 'missing hotspot' was scored zero. In each case, the mean MVD per section was calculated. Repeat analysis $(n=4)$ by the same operator generated coefficients of variation of 2\% (low MVD) and 13\% (high MVD).

Immunohistochemistry for Ki-67. Mouse monoclonal anti-Ki-67 (clone MIB1) antibody (Dako, Dako UK Ltd) was used to identify proliferating malignant epithelial cells by immunohistochemistry as described above except that slides were heated for $2 \mathrm{~min}$ in antigen retrieval buffer and antigen unmasking solution (both from Vector Laboratories, CA, USA) using a pressure cooker. A 1:50 dilution of primary antibody in Invitrogen antibody diluent was incubated with slides for $1 \mathrm{~h}$ at room temperature.

Measurement of the tumour proliferation index. Each section was scanned at $\times 100$ magnification and a high-power field $\left(\times 400 ; 0.07 \mathrm{~mm}^{2}\right)$ containing all tumour tissue and a minimum 200 malignant epithelial cells was chosen. If a slide did not fit these criteria, it was excluded from the analysis. The total number of stained and unstained epithelial cells was counted and the \% proliferation index (PI) was calculated as the number of positive cells divided by the total number of epithelial cells.

Microsatellite instability testing. MSI analysis was carried out by the Northern Molecular Genetics Service (Centre for Life, Newcastle Upon Tyne, UK) on $2 \times 10 \mu \mathrm{m}$ thick curls from each tumour block using the Promega MSI analysis system v1.2 (Promega UK, Southampton, UK), which analyses five mononucleotide repeat markers (BAT-25, BAT-26, NR-21, NR-24 and MONO-27). MSIhigh was defined as alteration in $\geqslant 2$ markers. 
Table 1. Characteristics of screen-detected and interval CRCs

\begin{tabular}{|c|c|c|c|}
\hline & $\begin{array}{l}\text { Screen-detected CRC } \\
(n=43)\end{array}$ & $\begin{array}{c}\text { Interval CRC } \\
(n=28)\end{array}$ & $P$-value \\
\hline $\begin{array}{l}\text { Site (number (\%)) } \\
\text { Distal to splenic flexure } \\
\text { Proximal to splenic flexure }\end{array}$ & $\begin{array}{l}29(67) \\
14(33)\end{array}$ & $\begin{array}{l}16(57) \\
12(43)\end{array}$ & 0.38 \\
\hline Size (maximum dimension in $\mathrm{mm}$, mean (range)) & $36(11-70)$ & $44(18-80)$ & 0.02 \\
\hline Ulcerating morphology (number (\%)) & $20(56)$ & 11 (44) & 0.17 \\
\hline $\begin{array}{l}\text { Dukes' stage (number }(\%))^{\mathbf{b}} \\
\text { A } \\
\text { B } \\
\text { C }\end{array}$ & $\begin{array}{r}5(12) \\
16(39) \\
20(49)\end{array}$ & $\begin{array}{r}6(21) \\
8(29) \\
14(50)\end{array}$ & 0.73 \\
\hline $\begin{array}{l}\text { Differentiation grade (number }(\%))^{c} \\
\text { Well } \\
\text { Moderate } \\
\text { Poor }\end{array}$ & $\begin{aligned} 7(16) \\
32(75) \\
4(9)\end{aligned}$ & $\begin{array}{r}7(25) \\
16(56) \\
5(19)\end{array}$ & 0.54 \\
\hline Extramural venous invasion (yes/no (\%)) & $4(10) / 37(90)$ & $11(42) / 15(58)$ & 0.005 \\
\hline
\end{tabular}

Statistical analysis. Student's $t$-test was used to compare continuous variables between the two CRC groups. The $\chi^{2}$-test or Fisher's exact test was used to compare categorical variables between the two groups. All analyses were performed using Stata v13.0 (StataCorp, College Station, TX, USA).

\section{RESULTS}

Archival tissue and the accompanying histopathology report for 98 CRCs were collected from three NORCCAG sites. Two cases were excluded on the basis that one was a secondary neoplasm from a non-CRC primary cancer and another case had multiple primary CRCs. Another archival CRC specimen was excluded as it was a duplicate of one of the CRC specimens. We next excluded 'polyp cancers' $(n=24)$ in which the primary tissue was adenoma, from which a focus of cancer had developed (exclusively screen-detected lesions), on the basis that the pathology and bleeding behaviour of a benign adenoma is likely to be different to an established malignant CRC, thus confounding comparison of biomarkers of tumour growth and bleeding tendency. Therefore, we analysed 71 non-polyp (screen detected $n=43$; interval $n=28$ ) CRCs.

Clinico-pathological characteristics of screen-detected and interval CRCs are described in Table 1. Interval CRCs were larger $(P=0.02)$ and were more likely to exhibit histological venous invasion $(P=0.005)$ than screen-detected tumours, although tumours were well matched for Dukes' stage (Table 1). The two groups were well matched regarding tumour location and histological differentiation grade, with $67 \%$ and $57 \%$ of tumours located distal to the splenic flexure in screen-detected and interval CRCs, respectively $(P=0.38)$. We were particularly interested in the presence of ulceration in individual CRCs as this may relate to bleeding propensity. We did not detect any difference in the proportion of screen-detected (56\%) or interval CRCs (44\%) that were reported to exhibit ulcerating morphology in the histopathology report. Interval CRCs in colonoscopy screening and surveillance programmes have been reported to be more likely to exhibit MSI (Sawhney et al, 2006). In our study, we did not observe a significant difference in the proportion of MSI-high tumours between screen-detected (5\%) and interval (14\%) CRCs, although

\begin{tabular}{|c|c|c|c|}
\hline & $\begin{array}{l}\text { Screen-detected } \\
\text { CRC }(n=43)\end{array}$ & $\begin{array}{c}\text { Interval CRC } \\
(n=28)\end{array}$ & $P$-value \\
\hline $\begin{array}{l}\text { Microvessel density } \\
\text { (mean (s.d.): range) }\end{array}$ & $96(7): 0-203$ & $95(8): 32-188$ & 0.52 \\
\hline $\begin{array}{l}\text { Proliferation index } \\
(\text { mean (s.d.) : range) }\end{array}$ & $70.5(2.7): 30.7-93.1$ & $66.5(4.2): 15.1-92.5$ & 0.80 \\
\hline
\end{tabular}

there was a numerical excess of MSI-high tumours in the interval CRC group. However, our study was underpowered to detect a significant difference, perhaps related to the relatively small number of proximal CRCs in the interval CRC group.

There was a wide range of tumour MVD values in both the groups, which mirrors known heterogeneity in CRC MVD values (Wang et al, 2014) (Table 2 and Figure 1). There was no significant difference in MVD values between screen-detected and interval CRCs (Table 2 and Figures 1A-C) suggesting no difference in vascularity of the two tumour groups. Similarly, there was no difference in the tumour PI between screen-detected and interval CRCs (Table 2 and Figures 1D-F), with the mean PI value for both groups in concordance with previous data in primary CRCs (Fodor et al, 2012).

We were also interested to note other pathology present in the surgical CRC resection specimens including synchronous colorectal polyps. Interestingly, there was a significantly higher proportion (51\%) of screen-detected CRC resection specimens that contained at least one additional colorectal polyp compared with the corresponding interval CRC specimens (18\%; Table 1). Importantly, the length of colectomy specimens in each group was similar (Table 3). More detailed analysis of polyp characteristics determined that there were 44 polyps detected in 22 screendetected CRC resection specimens compared with eight polyps in five interval CRC resection specimens (Table 3). The histological type and multiplicity of polyps in the two groups were similar, although polyps detected in screen-detected CRC specimens were larger than interval CRC resection specimen counterparts $(P=0.07$; Table 3$)$. 


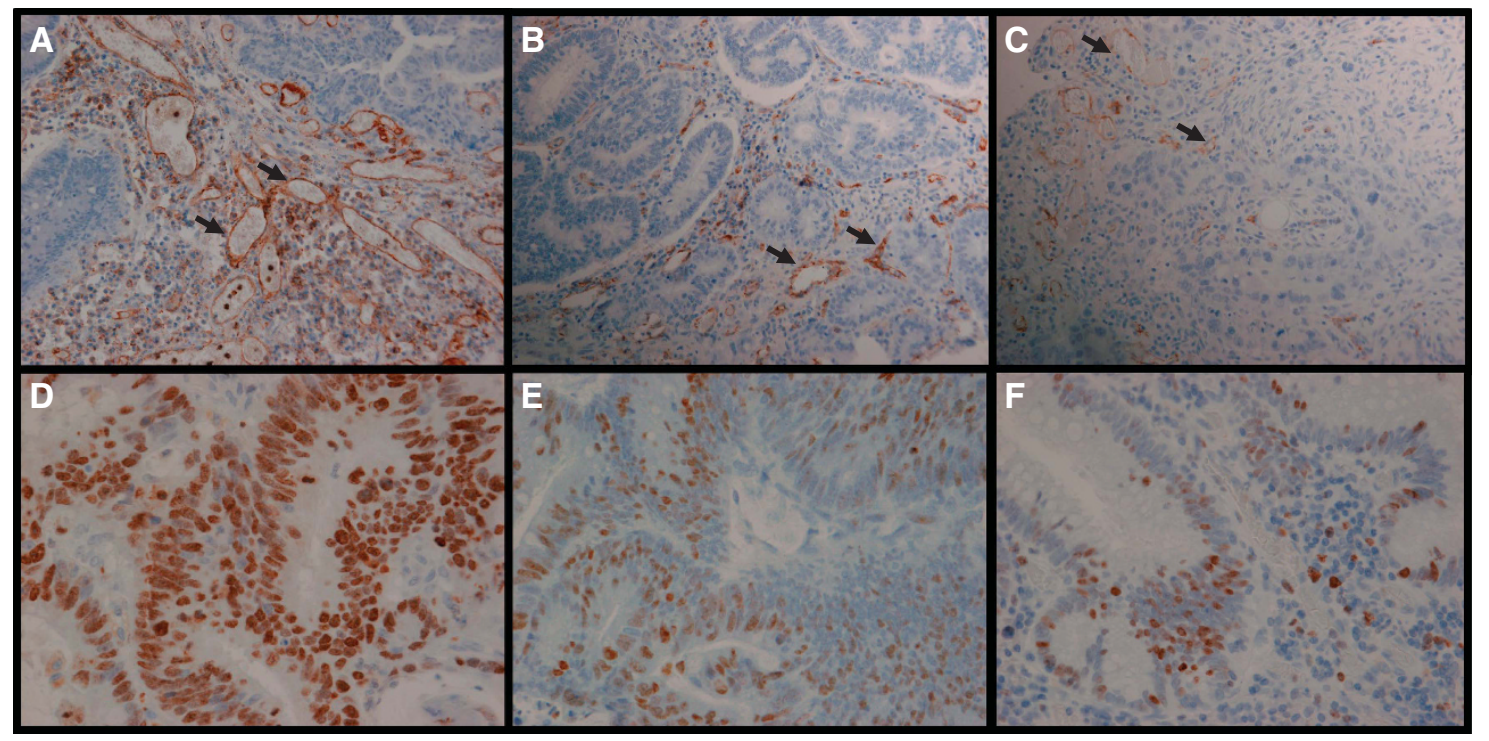

Figure 1. Immunohistochemistry for CD31 and Ki-67. (A-C) Examples of CRC 'hotspots' with high, medium and low MVD, respectively. Arrows highlight two of the vessels in each case. All images were captured at $\times 200$ magnification. D-F, examples of CRCs with a high, medium and low PI respectively. All images were captured at $\times 400$ magnification.

\begin{tabular}{|c|c|c|c|}
\hline & $\begin{array}{l}\text { Screen-detected CRC } \\
(n=22)\end{array}$ & $\begin{array}{c}\text { Interval CRC } \\
(n=5)\end{array}$ & $P$-value \\
\hline Total number of polyps & 44 & 8 & - \\
\hline Length of resection specimen $(\mathrm{mm} \text {, mean (range) })^{\mathbf{a}}$ & $356(150-1000)$ & $288(180-530)$ & 0.30 \\
\hline Size (mm, mean (range)) & $8(2-35) n=40^{b}$ & $5(3-6) n=7^{b}$ & 0.07 \\
\hline $\begin{array}{l}\text { Site (number }(\%)) \\
\text { Distal to splenic flexure } \\
\text { Proximal to splenic flexure }\end{array}$ & $\begin{array}{l}n=42^{b} \\
21(50) \\
21(50)\end{array}$ & $\begin{array}{l}3(38) \\
5(63)\end{array}$ & 0.70 \\
\hline $\begin{array}{l}\text { Histology (number (\%)) } \\
\text { TA } \\
\text { TVA or villous } \\
\text { Hyperplastic }\end{array}$ & $\begin{array}{l}6(14) \\
28(64) \\
10(23)\end{array}$ & $\begin{array}{l}n=7^{b} \\
0(0) \\
5(71) \\
2(29)\end{array}$ & 0.71 \\
\hline \multicolumn{4}{|c|}{$\begin{array}{l}\text { Abbreviations: } C R C=\text { colorectal cancer; } T A=\text { tubular adenoma; TVA }=\text { tubulovillous adenoma. Total number of additional polyps; screen-detected }(n=44) \text {, interval }(n=8) \text {. } \\
\text { a One (interval) case not known, one (screen-detected) case was colonoscopy finding, size taken as } 1000 . \\
\text { b } \\
{ }_{\text {Sample number quoted if any data missing. }}\end{array}$} \\
\hline
\end{tabular}

\section{DISCUSSION}

Understanding the biological characteristics of CRCs may improve the understanding of differences between screen-detected and interval cancers. Our previous study demonstrated improved outcomes in screen-detected patients with differences in biological characteristics provided as a possible explanation. In this study, we therefore sought to explore various characteristics of screendetected and interval CRCs. We did not demonstrate any difference in vascularity (measured by CD31-positive MVD) or tumour growth rate (measured by Ki-67 PI) between screendetected and interval 'non-polyp cancer' CRCs in the English BCSP.

It is a plausible hypothesis that differences in tumour bleeding propensity contribute to the detection of CRCs by the BCSP. We used immunohistochemistry for CD31 and vascular 'hotspot' counting as the most established methods for quantifying tumour vascularity (Wang et al, 2014). The degree of tumour surface erosion and ulceration may be critical to blood loss from a CRC (Hirano et al, 1996). However, we were unable to determine the degree of surface erosion and ulceration in archival fixed CRC tissue, which was only available retrospectively, often in multiple blocks. A frequent finding in CRC resection specimens is vascular congestion with blood engorgement of tumour vessels. However, this phenomenon is likely to be an artefact related to clamping of colorectal vasculature at surgery. Therefore, we did not attempt to measure vessel size or vascular congestion.

We did not provide any evidence that interval CRCs have a faster growth rate than screen-detected tumours based on the Ki67 PI. Therefore, the increased size and venous invasion observed in interval CRCs are most likely to be explained by longer lead time prior to diagnosis, rather than increased 'aggressiveness' of interval CRCs. The excess of interval CRCs in the proximal colon and higher proportion of MSI in interval CRCs in our study is consistent with previous reports and suggests that our CRC series is representative of screen-detected and interval CRCs from other screening programmes (Sawhney et al, 2006; Steele et al, 2012; Gill et al, 2014).

A provocative finding was the increased prevalence of polyps in screen-detected CRC resection specimens compared with interval $\mathrm{CRC}$ resection specimens. This is despite the fact that individuals undergoing colorectal surgery for a screen-detected CRC were more likely to have undergone colonoscopy and polypectomy than 
those presenting with a CRC following a negative gFOBt. This raises the question as to whether synchronous colorectal polyps contribute to luminal blood loss and gFOBt positivity, over and above that from established CRCs. Counter-intuitively, intestinal blood loss from CRCs is intermittent and surprisingly low as measured by ${ }^{51} \mathrm{Cr}$ loss, being only slightly greater than in patients with adenomatous polyps (Doran and Hardcastle, 1982; Herzog et al, 1982). Therefore, blood loss from synchronous polyps may provide a significant contribution to overall blood loss and performance of the low-sensitivity gFOBt. Alternatively, synchronous neoplasia may be a surrogate marker of increased 'field effect' mucosal microvascular blood content, which might contribute significantly to faecal blood loss in addition to that from neoplastic lesions (Roy et al, 2008). However, the number of synchronous polyps in each CRC group was relatively small, limiting the strength of any conclusion that can be drawn about a possible contribution of polyps to intestinal blood loss.

Independent studies have reported that the faecal haemoglobin concentration measured by the faecal immunochemical test is higher in CRC patients than those with colorectal adenomatous polyps and that faecal haemoglobin concentration correlates with tumour size and location (Ciatto et al, 2007; Digby et al, 2013). It will be important to perform a similar comparison of screendetected and interval CRCs in the proposed faecal immunochemical test-based screening programme in England. The quantitative faecal immunochemical test will allow a more detailed comparison of intestinal blood loss between patients with CRC with and without synchronous adenomatous polyps.

In conclusion, we did not detect any biological difference between screen-detected and interval CRCs in the English gFOBtbased BCSP, except for tumour size and venous invasion. This finding is consistent with low sensitivity of the gFOBt for CRC detection as the main driver of interval CRC (rather than rapid growth and/or reduced bleeding propensity) in the English BCSP. Differences in survival between screen and interval cancer patients are most likely explained by lead-time bias.

\section{ACKNOWLEDGEMENTS}

This work was funded by the South Tyneside NHS Trust Charitable Foundation and supported by the Northern Regional Endoscopy Group.

\section{CONFLICT OF INTEREST}

The authors declare no conflict of interest.

\section{REFERENCES}

Ciatto S, Martinelli F, Castiglione G, Mantellini P, Rubeca T, Grazzini G, Bonanomi AG, Confortini M, Zappa M (2007) Association of FOBTassessed faecal $\mathrm{Hb}$ content with colonic lesions detected in the Florence screening programme. Br J Cancer 96: 218-221.
Digby J, Fraser CG, Carey FA, McDonald PJ, Strachan JA, Diament RH, Balsitis M, Steele RJC (2013) Faecal haemoglobin concentration is related to severity of colorectal neoplasia. J Clin Pathol 66: 415-419.

Doran J, Hardcastle JD (1982) Bleeding patterns in colorectal cancer: the effect of aspirin and the implications for faecal occult blood testing. Br J Surg 69: 711-713.

Fodor IK, Hutchins GG, Espiritu C, Quirke P, Jubb AM (2012) Prognostic and predictive significance of proliferation in 867 colorectal cancers. J Clin Pathol 65: 989-995.

Gill M, Bramble M, Rees C, Lee T, Bradburn D, Mills S (2012) Comparison of screen-detected and interval colorectal cancers in the Bowel Cancer Screening Programme. Br J Cancer 107: 417-421.

Gill MD, Bramble MG, Hull MA, Mills SJ, Morris E, Bradburn DM, Bury Y, Parker CE, Lee TJW, Rees CJ (2014) Screen-detected colorectal cancers are associated with an improved outcome compared with stage-matched interval cancers. Br J Cancer 111: 2076-2081.

Herzog P, Holtermuller KH, Preiss J, Fischer J, Ewe K, Schreiber HJ, Berres M (1982) Fecal blood loss in patients with colonic polyps: a comparison of measurements with 51chromium-labelled erythrocytes and with the Haemoccult test. Gastroenterology 83: 957-962.

Hirano K, Uno Y, Munakata A, Yoshida Y (1996) Factors that influence bleeding in superficial-type colorectal tumors: study of endoscopic and histologic correlates. J Gastroenterol 31: 518-524.

Morris EJA, Whitehouse LE, Farrell T, Nickerson C, Thomas JD, Quirke P, Rutter MD, Rees C, Finan PJ, Wilkinson JR, Patnick J (2012) A retrospective observational study examining the characteristics and outcomes of tumours diagnosed within and without of the English NHS Bowel Cancer Screening Programme. Br J Cancer 107: 757-764.

Rajaganeshan R, Prasad R, Guillou PJ, Chalmers CR, Scott N, Sarkar R, Poston G, Jayne DG (2007) The influence of invasive growth pattern and microvessel density on prognosis in colorectal cancer and colorectal liver metastases. Br J Cancer 96: 1112-1117.

Roy HK, Gomes A, Turzhitsky V, Goldberg MJ, Rogers J, Ruderman S, Young KL, Kromine A, Brand RE, Jameel M, Vakil P, Hasabou N, Backman V (2008) Spectroscopic microvascular blood detection from endoscopically normal colonic mucosa: biomarker for neoplasia risk. Gastroenterology 135: 1069-1078.

Rubenstein JH, Enns R, Heidelbaugh J, Barkun A (2015) AGA Institute guideline on the diagnosis and management of Lynch syndrome. Gastroenterology 149: 777-782.

Sawhney MS, Farrar WD, Gudiseva S, Nelson DB, Lederle FA, Rector TS, Bond JH (2006) Microsatellite instability in interval colon cancers. Gastroenterology 131: 1700-1705.

Steele R, McClements P, Watling C, Libby G, Weller D, Brewster D, Black R, Carey FA, Fraser CG (2012) Interval cancers in a FOBT-based colorectal cancer population screening programme: implications for stage, gender and tumour site. Gut 61: 576-581.

Wang Y, Yao X, Ge J, Hu F, Zhao Y (2014) Can vascular endothelial growth factor and microvessel density be used as prognostic biomarkers for colorectal cancer? A systematic review and meta-analysis. ScientificWorldJournal 2014: 102736.

West N, Poullis A, Leicester R (2008) The NHS Bowel Cancer Screening Programme-a realistic approach with additional benefits. Colorectal Dis 10: $708-714$.

This work is published under the standard license to publish agreement. After 12 months the work will become freely available and the license terms will switch to a Creative Commons AttributionNonCommercial-Share Alike 4.0 Unported License. 